\title{
Geometric quantization on homogeneous spaces and the meaning of 'inequivalent' quantizations
}

\author{
M. A. Robson网 \\ Department of Applied Mathematics and Theoretical Physics, \\ University of Cambridge, Silver Street, \\ Cambridge, CB3 9EW, U. K.
}

\begin{abstract}
Consideration of the geometric quantization of the phase space of a particle in an external Yang-Mills field allows the results of the Mackey-Isham quantization procedure for homogeneous configuration spaces to be reinterpreted. In particular, a clear physical interpretation of the 'inequivalent' quantizations occurring in that procedure is given.
\end{abstract}

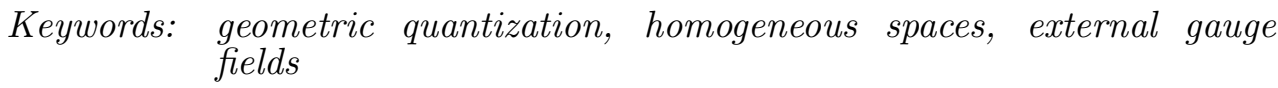

\section{Introduction}

Isham [1] has given a quantization procedure for a particle moving on a homogeneous configuration space, which was based upon the earlier work of Mackey [2]. Essentially quantization, in this approach, corresponds to finding irreducible unitary representations of a certain semidirect product group. We find that these representations occur via the geometric quantization of the phase space of a particle in an external Yang-Mills field. The symplectic formulation of such a phase space is described in section 2, while the results of geometric quantization, as applied to such spaces, is given in section 3. Note that our results are applicable for non-homogeneous configuration spaces and are thus presented for the general case. An outline of the Isham-Mackey quantization procedure is given in section 1 , while the comparison of the results of this method with those due to the geometric quantization approach is given in section 5 .

The mathematical techniques we use are standard symplectic geometry and Hilbert space representation theory. Full details of the results of the geometric quantization procedure given in section 03 may be found in [3] .

\footnotetext{
*E-mail: M.A.Robson@amtp.cam.ac.uk.

${ }^{\dagger}$ Supported by an E.P.S.R.C. studentship.
} 


\section{Symplectic formulation of the phase space}

We consider the case of a spin 0 particle moving on an arbitrary Riemannian manifold $Q$ in an external gauge field with gauge group $H$ (which we assume is compact). Mathematically, this is realised as a connection on a principal $H$-bundle over $Q$; the Yang-Mills field strength is identified with the local form of the curvature associated to the connection. Wong [i] introduced equations of motion for such a particle and it has been shown [5, 6, 7] that these are equivalent to the various mathematical formulations as given by the Kaluza-Klein formulation of Kerner [B] , and those of Sternberg [9] and Weinstein [7]. We use the symplectic formulation as detailed in [7, 5], since this is the most suited to the method of geometric quantization.

The right action of $H$ on $N$ lifts in a natural way to a symplectic right action on $T^{*} N$ with an equivariant momentum map $J: T^{*} N \rightarrow \mathbf{h}^{*}$, where $\mathbf{h}^{*}$ is the dual of the Lie algebra of $H$. The motion of the particle is described by an $H$-invariant Hamiltonian on $T^{*} N$ (the 'unconstrained' phase space). The metric on $N$ being induced by the connection and the metric on $Q$. The symmetry associated with the action of $H$ means that Marsden-Weinstein reduction can be used to identify the reduced phase space, $P_{\mu}$, of the system. Explicitly, $P_{\mu}=J^{-1}(\mu) / H_{\mu}$ for some $\mu \in \mathbf{h}^{*}$ [10]. Here $H_{\mu} \subset H$ is the isotropy group of the point $\mu$; the value of $\mu$ reflects which subset of $T^{*} N$ the particle is constrained to move in. Alternatively, the reduced phase space may be identified with $P_{\mathcal{O}_{\mu}}=J^{-1}\left(\mathcal{O}_{\mu}\right) / H \subset\left(T^{*} N\right) / H$, which is symplectically diffeomorphic to $P_{\mu}$ [11, 12]. Here $\mathcal{O}_{\mu} \subset \mathbf{h}^{*}$ is the coadjoint orbit containing the point $\mu \in \mathbf{h}^{*}$. Physically, $\mathcal{O}_{\mu}$, in its entirety, represents the charge of the particle. Thus, the possible reduced phase spaces of the particle are in one-to-one correspondence with its charge. Indeed, each $P_{\mathcal{O}_{\mu}}$ is in fact a symplectic leaf in the Poisson manifold $\left(T^{*} N\right) / H$, which inherits a Poisson bracket from the canonical one on $T^{*} N$.

The rôle of the connection is that it allows a factorization of $T^{*} N$ to be obtained, viz. $T^{*} N \simeq N^{\#} \times \mathbf{h}^{*}$ [5]. Here $N^{\#}$ is the pullback bundle of $N \rightarrow Q$ via the projection map $\pi: T^{*} Q \rightarrow Q$, and inherits a right action of $H$. In this trivialization the momentum map has the particularly simple form $J\left(\kappa_{n}, \nu\right)=\nu$, which means that we can take $P_{\mu}=N^{\#} / H_{\mu}$ and $P_{\mathcal{O}_{\mu}}=N^{\#} \times_{H} \mathcal{O}_{\mu}$ (i.e., $N^{\#} \times \mathcal{O}_{\mu}$ quotiented by the equivalence relation $\left.\left(\kappa_{n}, \nu\right) \sim\left(\kappa_{n} h, A d_{h}^{*} \nu\right)\right)$. Note that $N^{\#} / H=T^{*} Q$, so the connection allows a projection from $P_{\mathcal{O}_{\mu}}$ to $T^{*} Q$ to be defined. Considering $P_{\mathcal{O}_{\mu}}$ as the reduced phase space, then, as noted by Weinstein [7], until the connection is 
chosen the variables conjugate to position on $Q$ are inherently intertwined with the 'internal' variables associated to $\mathcal{O}_{\mu}$. Physically, this means without a connection we cannot separate the the particle's external momentum from its own 'position' and 'momentum' which is associated with the motion on the coadjoint orbit $\mathcal{O}_{\mu}$.

\section{Geometric quantization}

Notation. We will denote the projection map of a bundle $C$ with base space $X$ by $\pi_{C \rightarrow X}$.

The method of geometric quantization requires a complex line bundle, $B$ (the prequantum line bundle), over $P_{\mu}$ together with a connection on $B$ with curvature $\hbar^{-1} \sigma$, where $\sigma$ is the symplectic two-form on $P_{\mu}$. It is well known that not all symplectic manifolds admit such a line bundle. We find that the bundle $B$ exists provided that there is a representation, $\chi_{\mu}$, of $H_{\mu}$ into $\mathrm{U}(1)$ with gradient $i \hbar^{-1} \mu$ at $e \in H_{\mu}$. This is the same as Kostant's 13 formulation of the integrality condition for the quantization of the coadjoint orbit $\mathcal{O}_{\mu}$. Indeed, we shall see that the quantization of $P_{\mu}$ and $\mathcal{O}_{\mu}$ is closely connected.

Once the prequantum line bundle has been constructed, the next step is to choose a polarization of $P_{\mu}$. We construct a polarization on $P_{\mathcal{O}_{\mu}} \simeq P_{\mu}$ in the following manner. On $T^{*} N \simeq N^{\#} \times \mathbf{h}^{*}$ we choose the vertical polarization; this restricted to $N^{\#}$ gives an $H$-invariant distribution $P_{0}$ on $N^{\#}$. Recall that the group $H$ is assumed to be compact, which implies that $H_{\mu}$ is connected [14. Hence the coadjoint orbit $\mathcal{O}_{\mu}$ has a natural $H$-invariant positive polarization $P^{\mathcal{O}_{\mu}}$ [15. The direct sum of the polarization $P^{\mathcal{O}_{\mu}}$ on $\mathcal{O}_{\mu}$ and the distribution $P_{0}$ on $N^{\#}$ gives a new $H$-invariant distribution on $N^{\#} \times \mathcal{O}_{\mu}$ which projects down to give a polarization $P$ of $P_{\mathcal{O}_{\mu}}=N^{\#} \times_{H} \mathcal{O}_{\mu}$.

Attention is then restricted to the polarized sections of $B$. Crucially, we find that they can be realised as sections of the vector bundle $E=N \times_{H} \mathcal{H}_{\mu}$, where $(n, v) \sim\left(n h, \pi_{\mu}\left(h^{-1}\right) v\right)$ for $h \in H$. Here $\mathcal{H}_{\mu}$ is the Hilbert space that arises when the coadjoint orbit $\mathcal{O}_{\mu}$ is quantized. Specifically, it consists of functions $\phi: H \rightarrow \mathbb{C}$ which satisfy

$$
\phi\left(h_{\mu} h\right)=\chi_{\mu}\left(h_{\mu}\right) \phi(h) \quad \forall h_{\mu} \in H_{\mu}
$$

and are polarized with respect to the Kähler polarization on $\mathcal{O}_{\mu}$. (Here the $\phi$ are regarded as defining a section of a line bundle over $\mathcal{O}_{\mu}$.) An inner product on $\mathcal{H}_{\mu}$ is 
given by

$$
\left(\phi_{1}, \phi_{2}\right)_{\mathcal{H}_{\mu}}=\int_{H_{\mu} \backslash H} d\left([h]_{H_{\mu}}\right)\left\langle\phi_{1}(h), \phi_{2}(h)\right\rangle_{\mathbb{C}}
$$

The representation $\pi_{\mu}$ of $H$ is defined by

$$
\left(\pi_{\mu}\left(h^{\prime}\right) \phi\right)(h)=\phi\left(h h^{\prime}\right)
$$

Further it is possible to show that the representation $\pi_{\mu}$ is irreducible.

We identify the sections of $E$ with functions $\Psi: N \rightarrow \mathcal{H}_{\mu}$ satisfying $\Psi(n h)=$ $\pi_{\mu}\left(h^{-1}\right) \Psi(n)$ for all $h \in H$. Let $\eta$ be an $H$-invariant measure on $N$, which in turn determines a measure $\nu$ on $Q$. We can define an inner product on the sections of $E$ via

$$
\left(\Psi, \Psi^{\prime}\right)=\int_{Q} d \nu\left(\pi_{N \rightarrow Q}(n)\right)\left(\Psi(n), \Psi^{\prime}(n)\right)_{\mathcal{H}_{\mu}},
$$

and we restrict our attention to smooth functions $\Psi$ that have compact support. Let $C_{c}^{\infty}(Q)$ denote the subspace of smooth functions on $Q$ with compact support; then, following [16], we consider the induced unitary representation, $\pi^{\mu}$, of the semidirect product group Aut $N \ltimes C_{c}^{\infty}(Q)$ acting on the $\Psi^{\prime} s$ which is defined by

$$
\left(\pi^{\mu}(\phi, f) \Psi\right)(n)=\left(\frac{d \eta\left(\phi^{-1} n\right)}{d \eta(n)}\right)^{1 / 2} e^{-i \hbar^{-1} f \circ \pi_{N \rightarrow Q}(n)} \Psi\left(\phi^{-1} n\right) .
$$

Here $\phi \in$ Aut $N$ is an element of the group of automorphisms of $N$, i.e., diffeomorphisms of $N$ satisfying $\phi(n) h=\phi(n h)$. The group law on Aut $N \ltimes C_{c}^{\infty}(Q)$ being $\left(\phi_{1}, f_{1}\right) \cdot\left(\phi_{2}, f_{2}\right)=\left(\phi_{1} \circ \phi_{2}, f_{1}+f_{2} \circ \bar{\phi}_{1}^{-1}\right)$, where $\bar{\phi} \in$ Diff $Q$ denotes the projection of $\phi$ to $Q$. The representation $\pi^{\mu}$ is irreducible provided $N$ does not decompose into a disjoint union of two Aut $N$-invariant subsets both of which have positive $\eta$ measure. Note that in the case $N=G$, a Lie group, the [left] action of $G \subset$ Aut $G$ on $G$ is transitive and thus this condition is then automatically satisfied.

The action of the generators of $\pi^{\mu}$ are, in fact, the quantum operators corresponding to certain classical observables on $P_{\mathcal{O}_{\mu}}$. The correspondence is given via a momentum map, $J_{\mu}$, for the left action of Aut $N \ltimes C_{c}^{\infty}(Q)$ on $P_{\mathcal{O}_{\mu}}$. (This action is the standard one [14] of Diff $N \ltimes C^{\infty}(N)$ on $T^{*} N$ restricted to Aut $N \ltimes C_{c}^{\infty}(Q) \subset$ Diff $N \ltimes C^{\infty}(N)$, then restricted to acting on $J^{-1}\left(\mathcal{O}_{\mu}\right)$ and then dropped to give an action on $P_{\mathcal{O}_{\mu}}=J^{-1}\left(\mathcal{O}_{\mu}\right) / H$.) Denoting the Lie algebra of a group $\mathcal{G}$ by $\mathcal{L}(\mathcal{G})$, then the momentum map $J_{\mu}: P_{\mathcal{O}_{\mu}} \rightarrow \mathcal{L}\left(\text { Aut } N \ltimes C_{c}^{\infty}(Q)\right)^{*}$ is defined by

$$
\left\langle J_{\mu}\left[p_{n}\right]_{H},(A, f)\right\rangle=\left\langle p_{n}, A\right\rangle+\pi_{N \rightarrow Q}^{*} f(n) .
$$


Here $p_{n} \in J^{-1}\left(\mathcal{O}_{\mu}\right) \subset T^{*} N$ with $\pi_{T^{*} N \rightarrow N}\left(p_{n}\right)=n$, while $A$ is a [right] $H$-invariant vector field on $N$ regarded as an element in $\mathcal{L}$ (Aut $N)$ and $f \in C_{c}^{\infty}(Q)$, where the Lie algebra of $C_{c}^{\infty}(Q)$ has been identified with the group itself.

We can use the map $J_{\mu}$ to define a map $\hat{J}_{\mu}: \mathcal{L}\left(\right.$ Aut $\left.N \ltimes C^{\infty}(Q)\right) \rightarrow C^{\infty}\left(P_{\mathcal{O}_{\mu}}\right)$ via $\left(\hat{J}_{\mu}(A, f)\right)\left[p_{n}\right]_{H}=\left\langle J_{\mu}\left[p_{n}\right]_{H},(A, f)\right\rangle$ and it is this map which links observables on $P_{\mathcal{O}_{\mu}}$ to generators of $\pi^{\mu}$. Following the convention that the derived Lie algebra representation $d \tilde{\pi}$ of a representation $\tilde{\pi}$ is given by

$$
d \tilde{\pi}(A)=\left.i \frac{d}{d t} \tilde{\pi}\left(e^{t A}\right)\right|_{t=0}
$$

then, we find that the quantum operator corresponding to the classical observable $\hat{J}_{\mu}(A, f)$ is given by $\hbar d \pi^{\mu}(A, f)$. We express this in terms of a 'quantizing' map, $Q_{\hbar}$, which relates classical observables to quantum operators. We write

$$
Q_{\hbar}\left(\hat{J}_{\mu}(A, f)\right)=\hbar d \pi^{\mu}(A, f) .
$$

The operator $d \pi^{\mu}(A, f)$ acts on, and is essentially self-adjoint on, compactly supported sections of the vector bundle $E=N \times_{H} \mathcal{H}_{\mu}$.

The expression for $\hat{J}_{\mu}$ is slightly more illuminating if we use local coordinates for $P_{\mathcal{O}_{\mu}}=N^{\#} \times_{H} \mathcal{O}_{\mu}$. Now $N^{\#} / H=T^{*} Q$, and locally $P_{\mathcal{O}_{\mu}}$ is like $\left(N^{\#} / H\right) \times \mathcal{O}_{\mu}$. Thus, let $\left(h^{1}, \ldots, h^{d_{H}}, q^{d_{H}+1}, \ldots, q^{d_{N}}\right)$ be local coordinates on $N$, where $\left(q^{d_{H}+1}, \ldots, q^{d_{N}}\right)$ are coordinates on $Q$ and $\left(h^{1}, \ldots, h^{d_{H}}\right)$ are coordinates on the fibre $H$. Further, let $\left(p_{d_{H}+1}, \ldots, p_{d_{N}}\right)$ be the corresponding components of covectors on $T^{*} Q$. Then, locally, we can label a point $\left[p_{n}\right]_{H}$ in $N^{\#} \times{ }_{H} \mathcal{O}_{\mu}$ by $\left(q^{d_{H}+1}, \ldots, q^{d_{N}}, p_{d_{H}+1}, \ldots, p_{d_{N}}, \nu\right)$. Physically, the $q$ 's and $p$ 's represent the particle's external position and momentum respectively, while $\nu$ represents the internal 'position' and 'momentum'. We find

$$
\begin{aligned}
\hat{J}_{\mu}(A, f)\left[p_{n}\right]_{H}= & v^{\gamma}\left(q^{d_{H}+1}, \ldots, q^{d_{N}}\right) p_{\gamma}+\left\langle\nu, X\left(q^{d_{H}+1}, \ldots, q^{d_{N}}\right)\right\rangle \\
& +f\left(q^{d_{H}+1}, \ldots, q^{d_{N}}\right) .
\end{aligned}
$$

Here $v^{\gamma}$ are the components of the vector field $A$ on $N$ projected to $Q$ and $X \in \mathbf{h}$ is related to $A$ via the connection. The repeated index $\gamma$ is summed from $\operatorname{dim} H+1$ to $\operatorname{dim} N$. Equation (3.9) gives the local form of the observables which we can quantize via $Q_{\hbar}$. Further, if we require the quantization of a classical observable to give an essentially self-adjoint operator, then the image of $\hat{J}_{\mu}$ is indeed the complete set of observables that can be geometrically quantized in the normal manner. 


\section{Homogeneous configuration spaces}

When the bundle $N$ is a finite-dimensional Lie group $G$ (with $H \subset G$ ) the configuration space $Q=G / H$ is homogeneous. Isham [1] has considered quantization on such configuration spaces and in this section we briefly outline his scheme.

The core idea of Isham's approach, for a general configuration space $Q$, is to find a Lie group $\mathcal{G}$ which has a transitive Hamiltonian action on $T^{*} Q$ together with a momentum map $J_{I}: T^{*} Q \rightarrow \mathcal{L}(\mathcal{G})^{*}$. Quantization then corresponds to finding irreducible unitary representations of $\mathcal{G}$. For such a representation, $\pi_{I}$, the quantum

operator corresponding to the observable $\hat{J}_{I}(X) \in C^{\infty}\left(T^{*} Q\right)$ is given by $\hbar d \pi_{I}(X)$, i.e., $Q_{\hbar}\left(\hat{J}_{I}(X)\right)=\hbar d \pi_{I}(X)$. Here $X \in \mathcal{L}(\mathcal{G})$ and $\hat{J}_{I}: \mathcal{L}(\mathcal{G}) \rightarrow C^{\infty}\left(T^{*} Q\right)$ is defined in the same manner as $\hat{J}_{\mu}$ in section 3 .

Isham argues that the group $\mathcal{G}$ to use is any finite-dimensional subgroup of Diff $Q \ltimes C^{\infty}(Q) / \mathbb{R}$ (where $\mathbb{R}$ denotes the functions constant on $Q$ ) whose action is still transitive on $T^{*} Q$. In particular, for the homogeneous configuration space $Q=G / H$ Isham further motivates and justifies the choice of $\mathcal{G}=G \ltimes V^{*} \subset \operatorname{Diff} Q \ltimes C^{\infty}(Q) / \mathbb{R}$, where $V$ is a vector space which carries an almost faithful representation of $G$ and there is a $G$-orbit in $V$ that is diffeomorphic to $G / H$.

All the irreducible unitary representations of $\mathcal{G}$ can be constructed using Mackey theory; specifically, they are induced from irreducible unitary representations of subgroups $H^{\prime} \ltimes V^{*} \subset \mathcal{G}$ where $H^{\prime}$ is such that $G / H^{\prime} \simeq \Theta$, where $\Theta$ is a $G$-orbit in $V$. Now, in general, there will be more than one such irreducible unitary representation of $\mathcal{G}$ corresponding to each orbit. This immediately raises the first of two key questions, namely, which of these 'inequivalent' representations should be chosen? Secondly, in addition to the problem posed by the first question, what is the relevance of the representations corresponding to orbits $\Theta \simeq G / H^{\prime}$ where $H^{\prime} \nsucceq H$ ? We can answer these questions by considering the circumstances in which the geometric quantization approach generates such representations.

\section{The comparison}

Taking $N=G$ we see that Isham's phase space $T^{*} Q \simeq G^{\#} / H=P_{\mu=o} \subset\left(T^{*} G\right) / H$. Further, Isham's momentum map $J_{I}$ corresponds to the restriction of the momentum map $J_{\mu=0}$ of section 3 to $\mathcal{G} \subset$ Aut $N \ltimes C_{c}^{\infty}(Q)$. This illustrates the point that the geometric quantization approach considers $\mathcal{G}$ as a subgroup of Aut $N \ltimes C_{c}^{\infty}(Q)$ rather than Diff $Q \ltimes C_{c}^{\infty}(Q)$. 
We can split the irreducible unitary representations of $\mathcal{G}$ into two classes, those which arise from consideration of a $G$-orbit $\Theta \subset V$ where $\Theta \simeq G / H$ (the first class) and those from a $G$-orbit $\Theta^{\prime} \subset V$ where $\Theta^{\prime} \simeq G / H^{\prime}$ with $H \neq H^{\prime}$ (the second class). We can now compare Isham's approach with the geometric quantization one. Specifically, the representations $\pi^{\mu}$ we find are the same as those in Isham's first class. (Here we are restricting $\pi^{\mu}$ to $\mathcal{G}$.) Crucially, however, each of our representations corresponds, via $\mu$, to a different symplectic leaf, $P_{\mathcal{O}_{\mu}}$, in $\left(T^{*} G\right) / H$. Further, each symplectic leaf has a different momentum map and thus each of the different representations corresponds to a different classical system. In terms of the quantizing map $Q_{\hbar}$, Isham considers the phase space $G^{\#} / H \simeq T^{*} Q$ with

$$
Q_{\hbar}\left(\hat{J}_{\mu=0}(A, u)\right)=\hbar d \pi^{\mu}(A, u)
$$

where $(A, u) \in \mathcal{L}(\mathcal{G})^{*} \simeq \mathbf{g} \times V^{*}$. Note that it is not clear which representation $\pi^{\mu}$ is to be chosen on the right hand side. Whereas we have the phase space $G^{\#} \times_{H} \mathcal{O}_{\mu}$ with

$$
Q_{\hbar}\left(\hat{J}_{\mu}(A, u)\right)=\hbar d \pi^{\mu}(A, u)
$$

It is now clear that different representations of $\mathcal{G}$ correspond to different physical systems. In fact, for a particle moving in a Yang-Mills field, the different representations of $\mathcal{G}$ correspond to the different possible charges that the particle could have.

The representations of $\mathcal{G}$ in Isham's second class clearly correspond to the quantizations of constrained systems which have $H^{\prime}$ as the symmetry (gauge) group. In terms of a particle in a Yang-Mills field, these representations correspond to a particle on the configuration space $G / H^{\prime}$ where the internal charge couples to the gauge group $H^{\prime}$. Thus, they are unrelated to the original system.

In conclusion, we take issue with the common interpretation of the MackeyIsham quantization scheme as providing a number of inequivalent quantizations of a given classical system (viz. $T^{*}(G / H)$ ). Rather, each representation of the 'canonical

group' $\mathcal{G}$ (Isham), or of the system of imprimitivity defined by $G / H$ (Mackey), has been found to be the unique (geometric) quantization of a specific classical phase space.

\section{Acknowledgements}

The author would like to greatly thank N. P. Landsman for not only suggesting this line of research but also for his many helpful comments and suggestions. 


\section{References}

[1] C.J. Isham, Topological and global aspects of quantum theory, in: Relativity, Groups and Topology 2 (Les Houches Summer School 1983), eds. B.S. Dewitt and R. Stora (N. Holland, Amsterdam, 1984) p. 1059.

[2] G. Mackey, Induced Representations (Benjamin, New York, 1968).

[3] M.A. Robson, Preprint DAMTP 94-36 (1994).

[4] S.K. Wong, Nuova Cimento 65A (1970) 689.

[5] R. Montgomery, Letters in Math. Phys. 8 (1984) 59.

[6] J. Sniatycki, Hadronic J. 2 (1979) 642.

[7] A. Weinstein, Lett. Math. Phys. 2 (1978) 417.

[8] R. Kerner, Ann. Inst. Henri Poincaré 9 (1969) 143.

[9] S. Sternberg, Proc. Nat. Acad. Sci. 74 (1977) 5253.

[10] J.E. Marsden and J.E. Weinstein, Rep. Math. Phys. 5 (1974) 121.

[11] C.M. Marle, in: Differential Geometry and Relativity, eds. M. Cahen and M. Flato (D. Reidel, Boston, 1976).

[12] D. Kazhdan, B. Kostant, and S. Sternberg, Comm. Pure Appl. Math. 31 (1978) 481.

[13] B. Kostant, in: Lectures in modern analysis, volume 170 of Lecture notes in mathematics, ed. C.T. Taam (Springer, Berlin, 1970).

[14] V. Guillemin and S. Sternberg, Symplectic Techniques in Physics (Cambridge University Press, Cambridge, 1984).

[15] N.M.J. Woodhouse, Geometric Quantization (Clarendon Press, Oxford, second edition, 1992).

[16] N.P. Landsman, J. Geom. Phys. 12 (1993) 93. 